\title{
TAHITÍ HUBIERA PODIDO SER ESPAÑOLA
}

\section{TAHITI HAD BEEN SPANISH}

Guy Amaru: ANAYA

g.amaru@ozu.es

\section{CURRÍCULUM VITAE}

Francés nativo de Tahití. Es capitán de la Marina Mercante francesa. Pertenece a la sociedad francesa ANAYA compuesta de españoles en Francia y franceses amantes de la cultura española.

\section{RESUMEN}

Tahití es la isla más grande de la Polinesia Francesa, un territorio de ultramar francés al Sur del Océano Pacífico. La isla fue descubierta en 1606 por el marino y explorador español Pedro Fernández de Quirós. La Corona de España tomó posesión de la isla en 1772 para controlar la expansión de otras potencias y evangelizarla. La presencia española se mantuvo hasta 1776.

\section{PALABRAS CLAVE}

Tahití - España - Boenechea 


\section{ABSTRACT}

Tahiti is the largest island of French Polynesia, a French overseas territory in the South Pacific Ocean. The island was discovered in 1606 by Spanish sailor and explorer Pedro Fernandez de Quiros. The Crown of Spain took possession of the island in 1772 to control the spread of other powers and evangelize. The Spanish presence was maintained until 1776.

\section{KEY WORDS}

Tahiti - Spain - Boenechea

\section{ÍNDICE}

\section{Introducción}

2. La presencia española en Tahití (1772 - 1776)

2.1. Domingo de Boenechea - Primer viaje a Tahití (1772 - 1773)

2.2. Domingo de Boenechea - Segundo viaje a Tahití (1774 - 1775)

2.3. La misión española en Tautira (Diciembre 1774 - Noviembre 1775)

2.4. Abandono de la misión - fin de la presencia española en Tahití (1775)

3. Conclusión

4. Bibliografía 


\section{TEXTO:}

\section{Introducción}

Todos conocemos la isla de Tahití (Polinesia francesa), al menos por su reputación de lugar paradisíaco, el sentido de la hospitalidad de sus habitantes, la belleza de las "vahine" (mujeres), un sentimiento de la vida todo lo contrario de trágico, etc. Esta fama viene de los primeros navegadores que pisaron esa tierra a mediados del siglo XVIII, y se justifica perfectamente. Claro que las cosas han cambiado un poco, con los problemas que aportó la modernidad pero, junto con las otras islas del archipiélago, sigue siendo un país maravilloso.

Tahití está situada en el Centro/Sur del Océano Pacífico, en los $17^{\circ} 30^{\prime}$ de latitud sur, es decir, la misma que la frontera entre Perú y Chile. Dista de las costas de América del Sur unas 4.200 millas $(7.800 \mathrm{kms})$. Al Oeste, Nueva Zelanda se encuentra a 2.200 millas y Australia a 3.300. A pesar de esta situación tan aislada, no dejó de ser una escala imprescindible en las rutas marítimas (y hoy día aéreas) que cruzan el Pacífico.

Forma parte del inmenso triángulo maorí (polinesio), cuyos vértices son las Islas Hawaii al Norte, Nueva Zelanda al Suroeste y la Isla de Pascua (Rapa Nui) al Sureste. Los Polinesios son los habitantes de esta área, y sus dialectos, de orígen común, aún tienen muchas similitudes. Es el caso del idioma tahitiano, hablado en la casi mayoría del territorio llamado Polinesia francesa.

Francesa por los azares de la Historia. Pues, entre 1767, fecha de la primera llegada de los europeos (el inglés Wallis) a Tahití, y 1880, cuando se convirtió en colonia francesa, los ingleses e incluso los españoles se la disputaron. Véamos cuándo y cómo. 


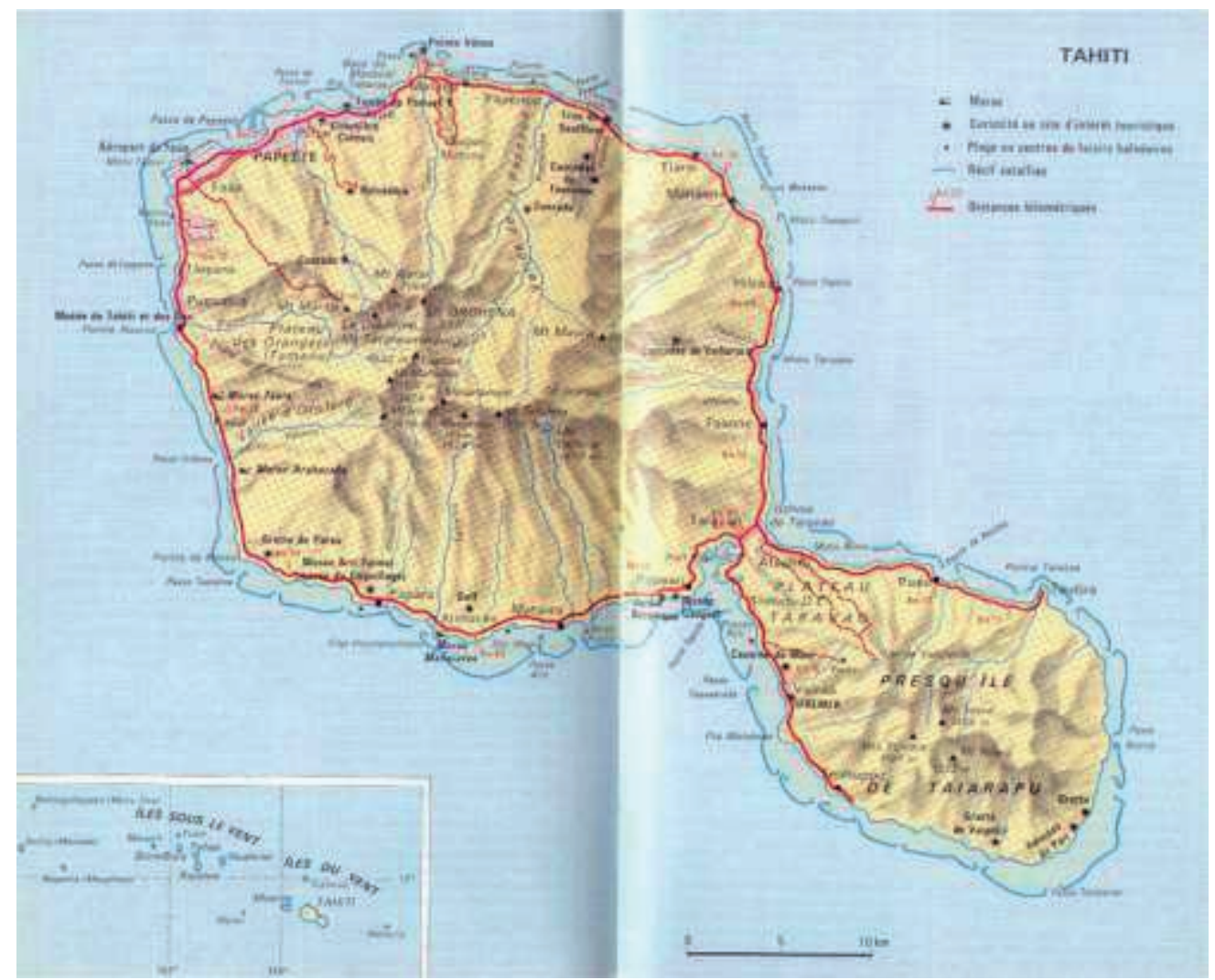

\section{La presencia española en Tahití (1772 - 1776)}

No hablaremos aquí de la breve escala que hicieron, en 1595, Álvaro de Mendaña y Pedro Fernández de Quirós en las islas que descubrieron y llamaron Marquesas. Ese archipiélago está situado al Norte de la actual Polinesia francesa, a 750 millas de Tahití.

Ya en 1770, el Virrey de Nueva Castilla (El Perú), Manuel Amat y Junyent, manda una expedición a la isla de Pascua, descubierta en 1722 por el holandés Roggeveen. La capitanea González de Haedo. Con sus dos barcos "San Lorenzo" y "Santa Rosalía", llega a Rapa Nui el 15 de noviembre de 1770 y toma posesión de la isla en nombre del Rey de España Carlos III. La llama Isla de San Carlos y establece su carta hidrográfica. Participa en la expedición un cierto Máximo Rodríguez, soldado de infantería de marina nacido en Perú, que volveremos a encontrar más adelante. Los 
barcos regresan al Callao el 29 de marzo de 1771, trás una escala en la isla de Chiloé, en Chile. El relato del viaje, trasmitido a Carlos III, pone de relieve la importancia de la isla para la seguridad del Perú y de Chile. Como consecuencia, el Rey manda instrucciones a Amat y Junyent para que envíe colonos y misioneros a Pascua.

\subsection{Domingo de Boenechea - Primer viaje a Tahití (1772 - 1773)}

Enterado del viaje del Inglés Cook a Tahití (1769), y sospechando un proyecto de colonización por parte de los ingleses, Amat y Junyent decide dar prioridad a Tahití, y sólo después cumplir las órdenes reales relativas a la Isla de Pascua.

Así pues, ordena una expedición a Tahití, bajo el mando de Domingo de Boenechea con Tomás Gayangos como ayudante. "El Aguila" zarpa del Callao el 26 de septiembre de 1772. A bordo está Máximo Rodríguez.

Boenechea es oriundo de Guetaria y Gayangos de Casa de la Reina (Logroño).

Llega "El Aguila" a Tahití el 12 de noviembre. Desembarcan unos oficiales y marineros con una lancha en la costa Este. Les acogen los tahitianos muy cordialmente. El 13, al intentar Boenechea entrar en la laguna, encalla "El Aguila". Afortunadamente, logran salvarlo y deciden trasladarse a la costa Norte de la península de Taiarapu, donde fondean frente al pueblo de Tautira, que llamarán Santísima Cruz, y donde se quedarán hasta su salida el 20 de diciembre. 


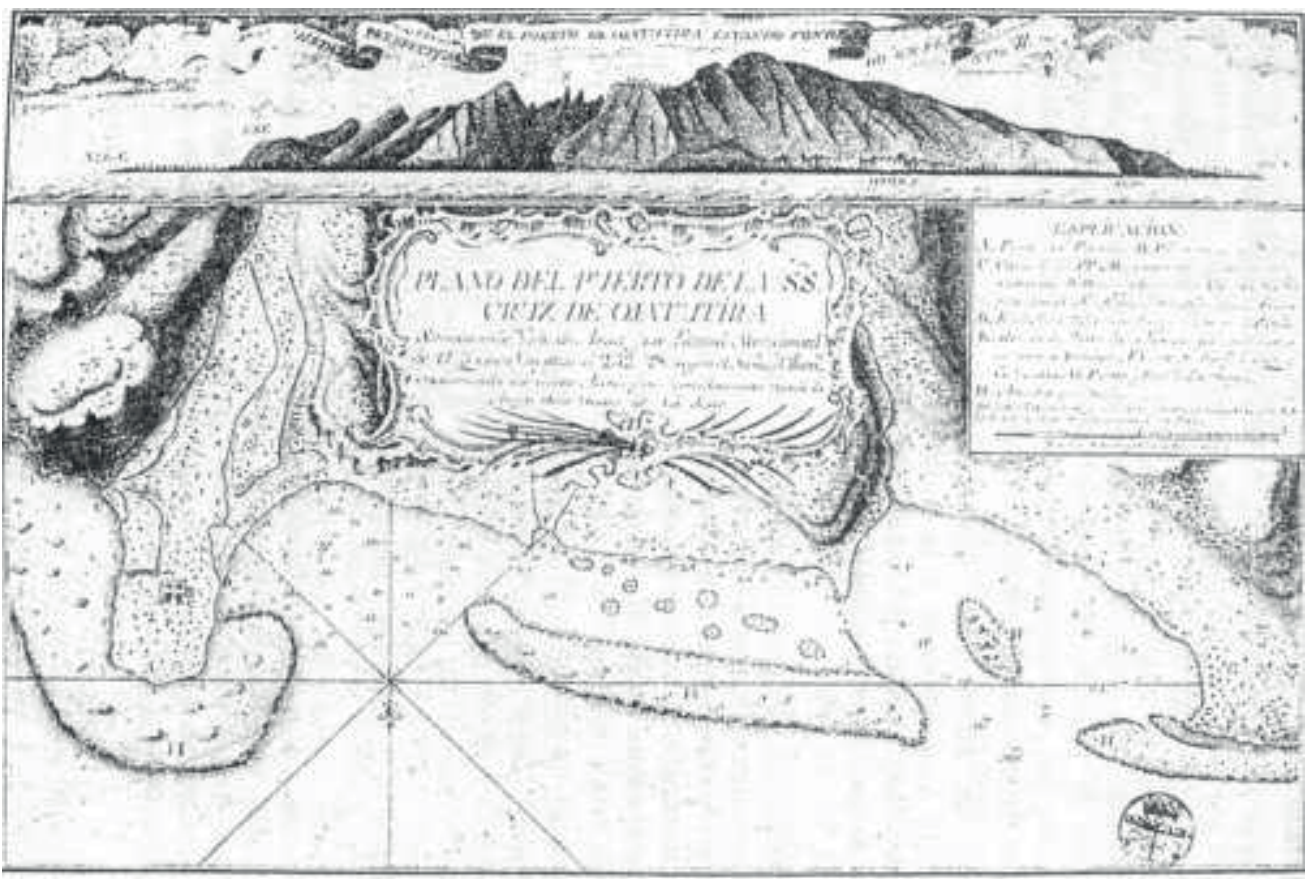

Plan du port de la Samsisima Cruz de Tautira, a Tahiti (Musée Nanal, Madrid).

Plano del Puerto de la Santísima Cruz. Siglo XVIII.

Imagen de "Les Espagnols à Tahiti". Publications de la Société des Océanistes, Paris 1995. 


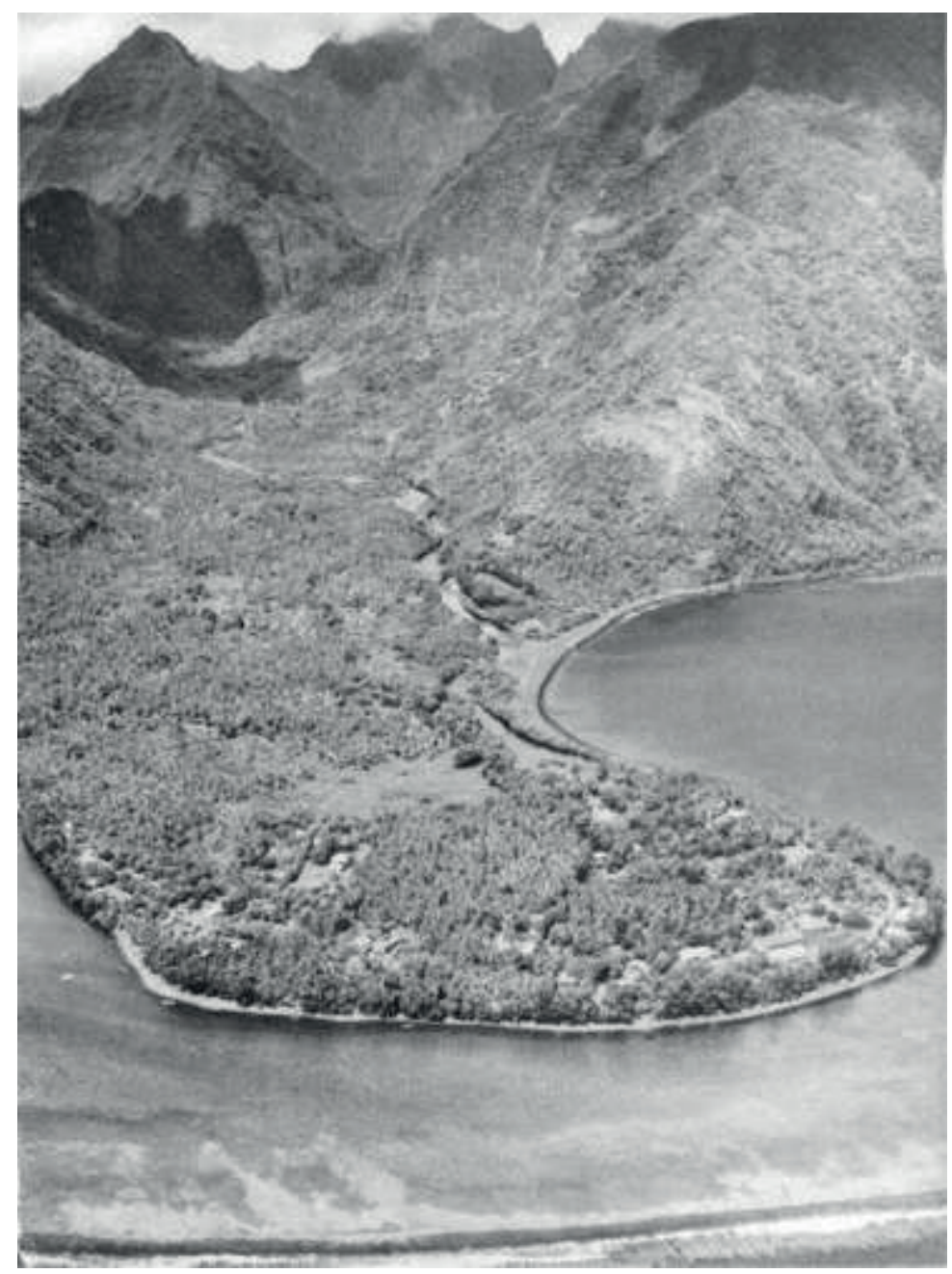

Foto aérea de Tautira.

Imagen de "Le Tahiti catholique", Patrick O’Reilly. Publications de la Société des Océanistes 1969.

Las órdenes de Boenechea son claras: entablar buenas relaciones con los indígenas, tratarlos bién, respetar sus pertenencias. Además, prohibe las relaciones sexuales con las mujeres, lo que les sorprenderá mucho, ya que para ellas eran cosas tan naturales y habituales que, con los Ingleses de Cook y los franceses de Bougainville, habían ocurrido muchas cosas....

Los Españoles llaman a la isla "Isla de Amat" y recogen todos los datos que pueden sobre las costumbres, la flora y la fauna, las posibilidades agrícolas, y también sobre 
los hechos y proyectos de los Ingleses. Comprueban que no hay colonización inglesa en Tahití y tampoco en la isla de Moorea que le hace frente. Gayangos, con una lancha, da la vuelta a la isla en cinco días y establece su carta y otros planos que hoy día podemos ver en el Museo Naval de Madrid. En cada una de sus etapas, los indígenas le acogen con alegría y muestras de amistad. Por su parte, Rodríguez profundiza en su conocimiento del idioma, partiendo de lo que ya había aprendido en la Isla de Pascua, donde se hablaba un dialecto muy parecido.

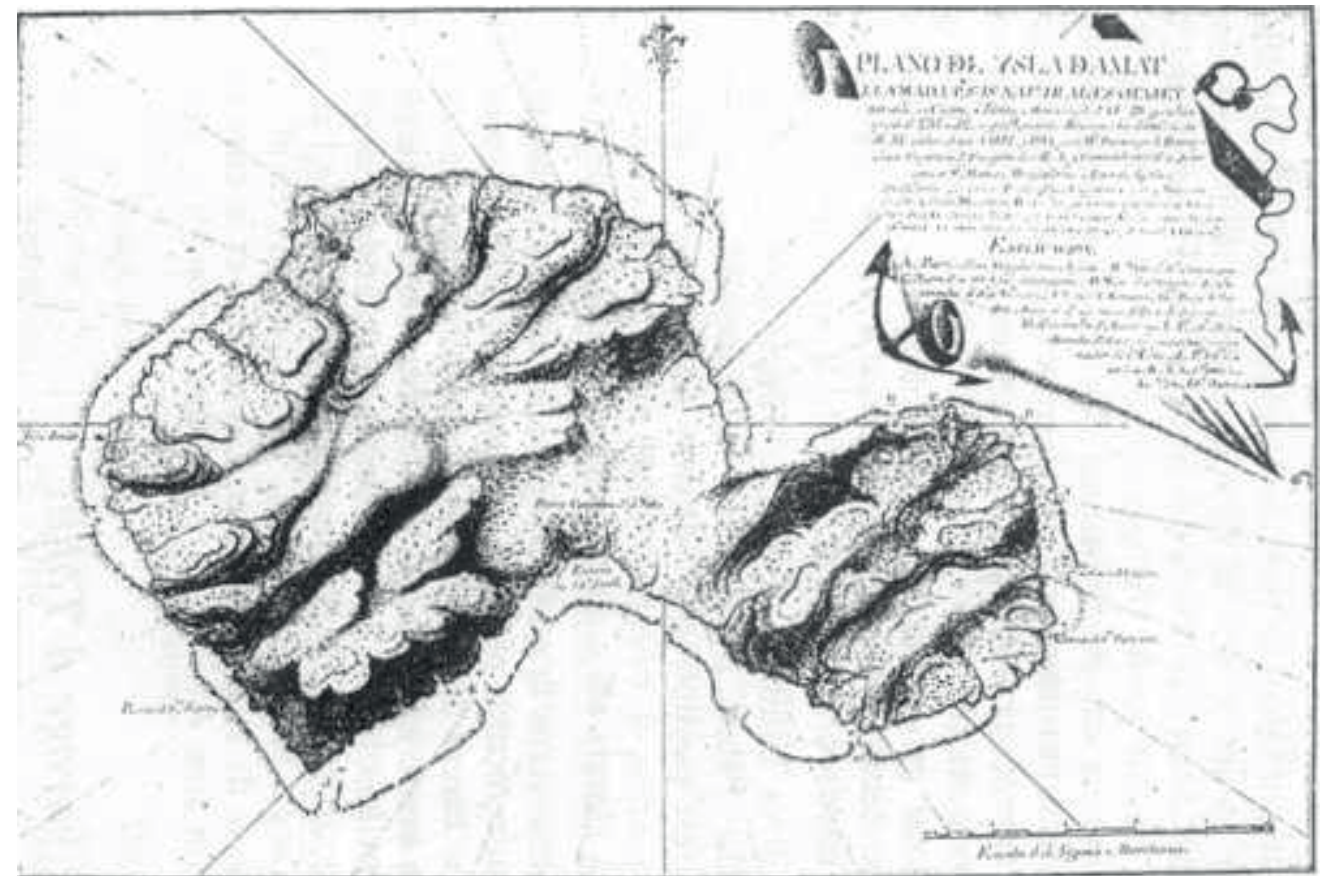

Carte de Iille de Tahiti, reconmue par lexpédition espagnole de Boenechea dans les années 1772 et 1774 (Musée Naval, Madrid).

Plano de la Isla de Amat. Siglo XVIII.

Imagen de "Les Espagnols à Tahiti". Publications de la Société des Océanistes, Paris 1995.

Al salir "El Aguila", el 20 de diciembre, cuatro tahitianos han aceptado hacer el viaje hasta el Perú, lo que Rodríguez aprovechará para perfeccionar su dominio del idioma. Llegan primero a Valparaíso de Chile, el día 21 de febrero de 1773, para unas 
reparaciones antes de dirigirse a la Isla de Pascua, a fin de cumplir las órdenes reales. En esta escala chilena muere uno de los tahitianos a causa de la gripe.

"El Aguila", debido a una avería grave, tiene que interrumpir su viaje a Pascua y regresa al Callao el 31 de mayo. Todo el material recogido en Tahití será presentado al Virrey, lo que le decide a preparar otra expedición. Por su parte, los tres tahitianos son alojados en una dependencia del palacio en Lima y educados como españoles. Uno fallece en septiembre de la viruela y los otros dos reciben bautismo, con gran pompa, en la Catedral, siendo sus padrinos personalidades del entorno del Virrey.

\subsection{Domingo de Boenechea - Segundo viaje a Tahití (1774 - 1775)}

En vista de los resultados del primer viaje, Amat y Junyent planea otro, con objetivos más ambiciosos: anexar la isla, someter los habitantes a la Corona y evangelizarlos; además, explorar las islas cercanas a Tahití.

No vacila en los medios para alcanzar el reto: la expedición estará bajo el mando de Boenechea y constará de dos barcos, el "Aguila" otra vez, acompañado por el "Jupiter", cuyo capitán será José Andía y Varela. Tiene previsto fundar un asentamiento, más bien una misión, empezando con dos sacerdotes franciscanos, un intérprete y un ayudante. Serán respectivamente Jerónimo Clota, oriundo de Olot (Gerona), Narciso González, de Montemolín (Badajoz), Máximo Rodríguez, nombrado oficialmente intérprete, y un grumete gallego. Cargarán herramientas, semillas, ganado, y una casa prefabricada para los cuatro. También utilizarán un "Diccionario" básico que Rodríguez redactó en Lima, ayudado por los Tahitianos, y un "Interrogatorio" de cien preguntas sobre varios asuntos, futuro marco del informe final sobre la estancia. (Copias de aquellos dos documentos aún existen en el Archivo General de Indias). Los dos tahitianos volverán a su isla, y deberán actuar de intermediarios entre los españoles y la población autóctona. 
Los barcos zarpan del Callao el día 20 de septiembre de 1774. El 5 de octubre, son separados por un temporal y el "Júpiter" llega a Tahití una semana antes que el "Aguila", el día 8 de noviembre. Han reconocido o descubierto una quincena de islas en los archipiélagos de Tuamotu, Islas a Sotavento y luego Australes.

Es de notar que Cook, en su segundo viaje al Pacífico, arribó a Tahití dos veces, en agosto de 1773 y en abril de 1774, pero sólo con objetivos científicos.

Tras reconocer unos puertos y bahías, los españoles fondean en Tautira, bahía y pueblo que ya conocen y que les parece más seguro e idóneo para sus planes. Entablan buenas relaciones con el jefe más importante de Tahití (o rey), llamado Tu, y el de la península, Vehiatua. Éste acepta que la misión se instale y que los frailes elijan un terreno que les convenga en la aldea. A finales de noviembre delimitan el solar y empiezan la construcción de la casa, en la que los frailes se instalan un mes más tarde.

El uno de enero de 1775, con gran solemnidad, erigen una cruz frente a la casamisión, con referencia al Cristo, al Rey Carlos III y la fecha 1774. Y se celebra la primera misa.

El día 5, en otra ceremonia con gran pompa, se establece el tratado conocido como "Las Capitulaciones de Oxatutira" (Tautira). El documento lo redacta Pedro Freyre de Andrade, secretario del "Aguila" (se halla en el Archivo General de Indias en Sevilla). Lo leen, con traducción de Rodríguez, en presencia de Boenechea y sus oficiales, a los dos jefes y a sus subditos que aceptan su contenido, a saber: reconocer la soberanía del Rey de España, aceptar la estancia de los cuatro españoles, protegerlos, suministrar todo lo que necesiten... Por otra parte, España se compromete en enviar barcos de manera regular, protegerlos de sus enemigos, proveer instrucción, 
abastecer en herramientas... Lo extraño es que el documento no tiene ninguna firma, ya sea de los contratantes, ya de los testigos.

Boenechea, cuya salud ya se había debilitado, muere el 26 de enero. El 27, en otra ceremonía solemne, lo entierran frente a la casa-misión, con su uniforme y con su espada, en presencia de numerosos indígenas curiosos y asombrados por los ritos funerarios de los extranjeros.

Al día siguiente, bajo mando de Gayangos, los dos barcos salen de Tahití rumbo al Callao, donde llegan en los primeros días de abril. Han dejado atrás a los cuatro miembros de la colonia española.

\subsection{La misión española en Tautira (Diciembre 1774 - Noviembre 1775)}

Desgraciadamente, no podemos decir que fuera un éxito.

Ante todo, y a pesar de su aislamiento, los cuatro no supieron mantener la paz entre sí, todo lo contrario. Como la jerarquía no había sido claramente determinada, los padres exigían de los demás mucho más de lo razonable, como si fueran criadas. El grumete, con continua mala leche, obedecía a regañadientes hasta que, de riña en riña, se pelearon a puñetazos. En cuanto a Máximo Rodríguez, se prevalía de su función de intérprete y de su misíon (aunque no oficialmente formulada) de estudio del país, para huir de las táreas domésticas. De tal manera que, muy a menudo, se marchaba de la casa-misión.

Lo mismo con los indígenas. Sí, las relaciones con Vehiatua y su familia fueron buenas, gracias, sobre todo, al jefe que siempre quiso cumplir sus obligaciones redactadas en las Capitulaciones, y a Rodríguez que se convirtió en el amigo de su hijo. Pero los españoles que confiaban mucho en los dos indígenas bautizados, no 
tardaron en quedar decepcionados porque, a los pocos días de su retorno, éstos se quitaron la ropa europea, olvidaron la educación cristiana e intrigaron contra los sacerdotes. Más grave, con los lugareños cada día occurían desacuerdos, riñas, hasta peleas graves, por motivos fútiles, por culpa, fundamentalmente, del abismo cultural entre los misioneros y los tahitianos.

En consecuencia, no es de extrañar que en un ambiente tal malo, los religiosos se aislaran en la casa-misión, renunciaran a todo intento serio de evangelización y esperaran la vuelta al Perú con gran ansiedad, a veces temiendo por su vida.

Afortunadamente, Máximo Rodríguez sería mucho más activo. Este limeño, nacido hacia el 1750, había viajado a España entre 1767 y 1769 como soldado de marina. Sus actuaciones y sus escritos demuestran un espíritu abierto, curioso, metódico y objetivo. Da dos veces la vuelta a la isla en piragua, ayudado por gente de Vehiatua. Parándose en las tierras de Tu, el mayor jefe de la isla, éste le confía un gran cuenco (umete) de piedra negra muy dura (dolerita), de unos 150 kilos, como regalo al Rey Carlos III. Ese objeto único venía del gran templo (marae) de Taputapuatea, en la costa oeste de la isla y podía servir para ritos religiosos. Llegará a España en 1788 y, hoy día, está conservado en el Museo Nacional de Antropología en Madrid. 


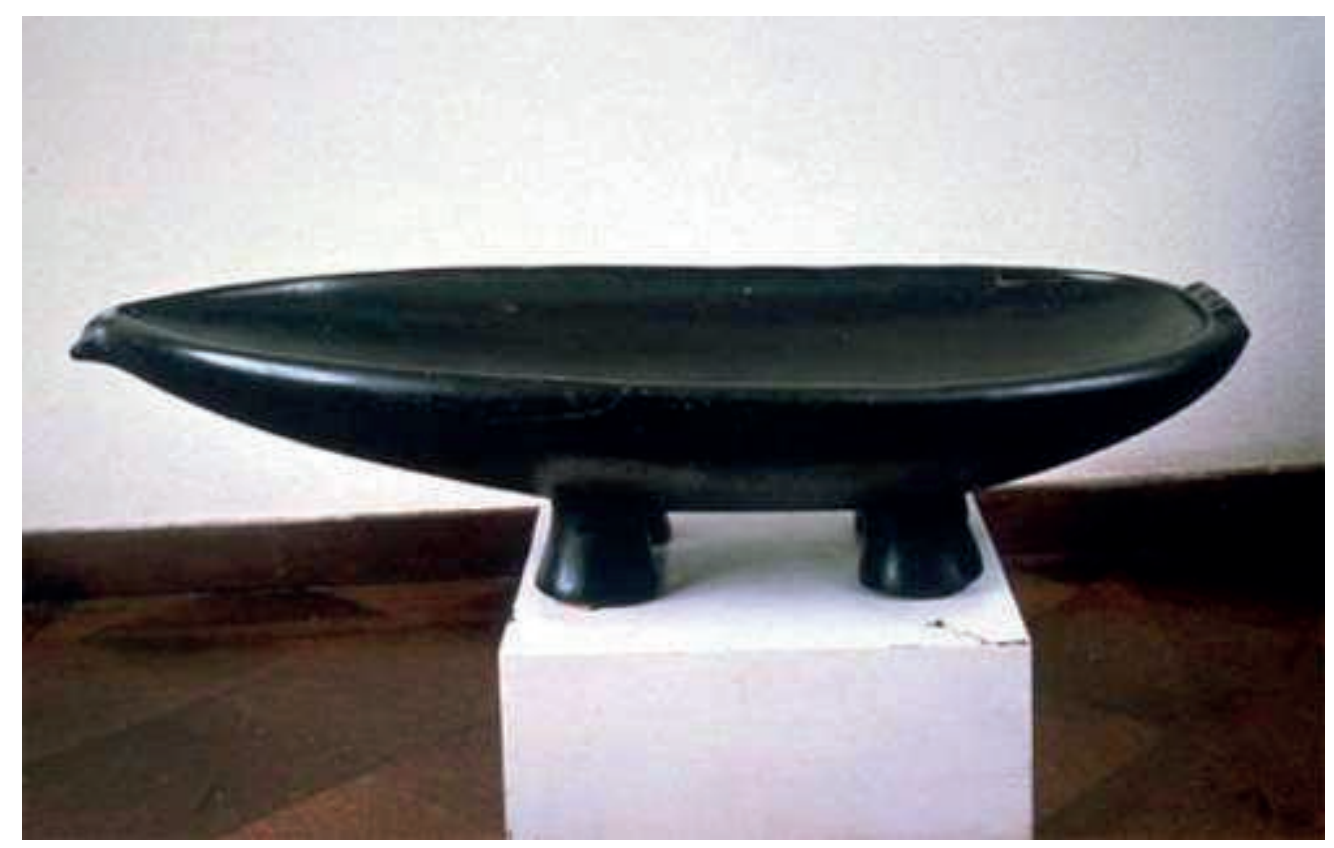

Cuenco de dolerita.

WEB del Museo Nacional de Antropología de Madrid.

Rodríguez aprovecha su larga estancia para redactar varias obras, entre las cuales un "Diario" completo y detallado de los 12 meses que duró la Misión. Representa el escrito más largo y antiguo sobre la manera de vivir de los tahitianos del siglo XVIII y sus costumbres. Por eso, tiene valor histórico inestimable. De las cuatro o cinco copias del "Diario", quedan una en la Biblioteca de la Royal Geographic Society en Londres, de 189 hojas recto-verso, y otra en la Biblioteca Nacional (Departamento de los mapas y planos) en París. Los primeros ejemplares, destinados uno al virrey Manuel de Amat, en 1776, y otro al virrey Teodoro de Croix, en 1788, para el Ministro de Marina Antonio Valdés y luego al Rey Carlos III (junto con el cuenco de dolerita) han desaparecido.

Desaparecieron también:

un "Vocabulario" tahitiano-español, con nociones de gramática.

un "Extracto", especialmente dedicado a sus observaciones sobre los ritos y las costumbres de los indígenas. 
En 1788, redacta un "Memorial" que describe su carrera y un "Prólogo" para contestar a Cook quien le acusaba en la "Relación de su tercer viaje" de haber denigrado a los ingleses ante los jefes tahitianos. Esos dos documentos estaban adjuntos al "Diario" destinado al virrey de Croix.

Es de notar también que el padre Clota redactó un "Memorial" de sólo algunas páginas, en el que relata, casi únicamente, las dificultades cotidianas de los dos sacerdotes: robos de gallinas, mala salud del padre Narciso y otras quejas. También hay algo más interesante sobre la muerte de Vehiatua y los ritos funerarios tahitianos, vistos a través del prisma de su educación católica. Nada o muy pocas cosas sobre sus intentos de evangelización, ni sobre la apostasía de los dos tahitianos.

Por fín, tenemos también otros relatos, no de la Misión, sino del viaje del "Aguila", escritos por navegantes, entre los cuales "el Diario de Navegación" de Gayangos y "el Relato de viaje" de José de Andía y Varela, capitán del "Jupiter".

\subsection{Abandono de la misión - fin de la presencia española en Tahití (1775)}

Tras el retorno de Gayangos al Callao, en abril de 1775, el informe de Manuel de Amat al Rey es muy positivo cuanto a las medidas que ha iniciado en Tahití. Propone seguir la experiencia y desarrollar la presencia española. Pero Madrid no va a respaldarlo. Porque eso no cabe con la política de despotismo ilustrado de Carlos III, que ya ha puesto en marcha medidas antireligiosas en España y en el Imperio: expulsión de los Jesuitas y luego supresión de la Companía de Jesús, coartación de los poderes de la Inquisición, etc. Además, se está acercando una nueva guerra contra Inglaterra. Así que manda sustituir a Amat por Manuel de Guirior, y luego por Teodoro de Croix. 
Pero al enterarse de los preparativos de Cook para su tercer viaje, Amat toma la iniciativa de otra expedición del "Águila" a Tahití. Lo capitaneará Juán Cayetano de Lángara. Sus órdenes son, según la "Relación de Gobierno" de Amat, asegurarse del estado de salud de los cuatro españoles y cumplir lo que los Franciscanos decidan, a saber, quedarse en Tahití y reforzar la Misión, o abandonarla definitivamente. Parece que, ya antes de que salga "El Aguila", la decisión de renunciar está hecha, porque el barco zarpará el 27 de septiembre de 1775 sin que hayan previsto nadie ni nada para reforzar la Misión.

En cuanto "El Aguila" llega a Tautira el 3 de noviembre, los dos sacerdotes exigen su regreso al Perú. Lángara intenta influir para que sigan, pero sin éxito. Por su parte, exige de los religiosos que expliquen por escrito sus motivos. Así, la escala va a durar muy poco. Los españoles se despiden del gran jefe $\mathrm{Tu}$ y recogen lo que pueden de sus pertenencias, dejando a los tahitianos las casas y unos cuantos animales.

El 12 de noviembre de 1775, "El Aguila" se hace a la mar rumbo al Callao. Manuel de Amat en su informe será muy crítico con los dos franciscanos, sin embargo se deshace en elogios hacia Rodríguez por su trabajo.

\section{Conclusión}

Las relaciones entre Perú y Tahití se reanudarán sólo un siglo más tarde, con viajes comerciales. Quedan hoy día muy escasas, casi inexistentes. Sí, Chile ha reanudado vinculos con Tahití, con su linea aérea entre Santiago, la Isla de Pascua y Papeete.

En memoria de ese breve episodio de historia común entre España y Tahití, se levantó una cruz en la aldea de Tautira, con la inscripción de Boenechea. En 1995 tuvo lugar un acto comemorativo por los 220 años de la primera misa católica en este mismo lugar, con presencia de personalidades religiosas y civiles tahitianas y españolas, y apoyo material del Museo Naval de Madrid. 


\section{Bibliografía}

"Les Espagnols à Tahiti". Publications de la Société des Océanistes, Paris 1995. Traducido al francés y anotado por Horacio Belçaguy, según "El Diario de Máximo Rodríguez" editado por Francisco Mellén Blanco a partir del manuscrito de Londres.

"Le Tahiti catholique", Patrick O’Reilly. Publications de la Société des Océanistes 1969. 